\title{
Stronger Lagrangian bounds by use of slack variables with applications to machine scheduling problems
}

\author{
Han Hoogeveen, Princeton University, U.S.A. \\ Steef van de Velde, University of Twente, The Netherlands
}

The basic idea behind Lagrangian relaxation is to see an NP-hard problem as an 'easy-to-solve' problem complicated by a number of 'nasty' side constraints. We show that reformulating nasty inequality constraints as equalities by using slack variables leads to stronger bounds. The improvement proceeds by computing a bound on the associated so-called slack variable problem. The trick is widely applicable, but we focus on machine scheduling problems, including the single-machine problem of minimizing total weighted completion time subject to precedence constraints, the two-machine flow-shop problem of minimizing total completion time, the single-machine problem of minimizing total weighted tardiness, and the flow-shop problem of minimizing total completion time with batch machines. We derive the slack variable problem for each of these problems and point out how lower bounds can be computed for it. For each application we obtain promising computational results in the sense that a significant improvement of the Lagrangian bound can be achieved relative to the extra needed to compute a lower bound on the slack variable problem. 